\title{
B-Scan Ophthalmic Ultrasonography of Retinal Detachment
}

\author{
Uduma $\mathrm{Fu}^{1 *}$, Akpan $\mathrm{Si}^{2}$, Megbelayin $\mathrm{E}^{2}$ and Abraham $\mathrm{E}^{2}$ \\ ${ }^{1}$ Department of Radiology, University of Uyo, Nigeria \\ ${ }^{2}$ Department of Ophthalmology, University of Uyo, Nigeria \\ *Corresponding author: Felix U Uduma, Department of Radiology, Faculty of Clinical Sciences, University of Uyo, Uyo, Nigeria. \\ To Cite This Article: Uduma Fu. B-Scan Ophthalmic Ultrasonography of Retinal Detachment. Am J Biomed Sci \& Res. 2019 - 4(5). AJBSR. \\ MS.ID.000827. DOI: 10.34297/AJBSR.2019.04.000827
}

Received: August 09, 2019 | Published: August 20, 2019

\begin{abstract}
Retinal detachment (RD) is categorized into rhegmatogenous, tractional or exudative. In as much as it can be detected using ophthalmoscopy, elucidation with B-scan ocular ultrasonography is not hindered by opaque medium. Besides, B-scan can even give an insight into RD aetiologies and associations.
\end{abstract}

Keywords: Retina; B-scan ultrasonography; Detachment; Vitreous

\section{Learning Points}

Grasping the diagnostic characteristics of retinal detachment using the seamless and non-invasive B-scan ophthalmic ultrasonography.

\section{Introduction}

\section{Background}

Retinal detachment (RD) is the separation of the neurosensory retina (anterior two-third) from the subjacent retinal pigment epithelium (RPE) \{posterior one-third\} [1].

\section{Classifications}

$\mathrm{RD}$ is sub-classified into rhegmatogenous, tractional and exudative [2,3]. The former is the commonest.

\section{Epidermiology}

Rhegmatogenous RD occurs in approximately 1 in 10000 people annually but it has geographical variations $[4,5]$.

\section{Pathophysiology}

Rhegmatogenous RD arises from rhegma, implying a rent. When there is retina break, fluid ingresses from the vitreous cavity to the sub-retinal potential space causing retinal detachment $[2,3,6]$. While anomalies in water transport across RPE cause exudative RD, tractional RD is due to scarring from formation of pre-retinal fibrovascular membrane.

\section{Risk Factors}

Risk factors are Aging, Diabetes, Vascular retinopathy (Eales's disease), trauma, sickle cell retinopathy, endophthalmitis, axial myopia, post-cataract surgery, family history, RD in one eye, genetic disorders like Marfan's syndrome, pre-existing retinal disease like coloboma choroid, retinoschisis, and acute retinal infections like CMV [2,3]. Posterior vitreous detachment (PVD) is the most common cause of retinal tears, a known cause of rhegmatogenous $\mathrm{RD}[4]$.

\section{Clinical Presentations}

Commonest presenting symptom is sudden, painless loss of vision or blurring of vision. Flashes and floaters may precede loss of vision pointing to PVD as the underlying aetiology of RD. Late presentation is seen in developing countries with involvement of macula in $90 \%$ of cases, retinal scarring and bilateral involvement [3].

\section{Diagnosis}

Though RD can be diagnosed with indirect ophthalmoscopy, B-scan ultrasonography has additional merits of identification of RD even in opaque medium. Possible lesion aetiology and associations like ocular tumour, floaters, PVD and choroidal detachment are also highlighted. 


\section{B-Scan Features}

A typical echogram of RD is a V-shaped echogenic intra-vitreous relatively mobile band that is anchored posteriorly to the optic disc but anteriorly to the ora serrata. When totally detached, RD appears as elevated convex bullae extending far into the vitreous from known attachment points [2]. If a concave membrane becomes a convex configuration on follow up, it is indicative of development of a rhegmatogenous element. [2] Tractional RD shows as a concave membrane with a varying extent of vitreous adhesions. Shifting fluid is seen in exudative RD. Often, the detachment may be confined to the macula in inflammatory or infective conditions [2].

In chronic $\mathrm{RD}$, bands become thickened, lowly reflective, less mobile and may harbour retinal cyst or reflective echoes of sub-retinal haemorrhage [2]. In cases of Proliferative Vitreous Retinopathy (PVR), the retina assumes a triangular or funnel shaped configuration which can range from open to tightly closed $[2,7]$.

\section{Management}

Retinal re-attachment surgery. This closes all retinal breaks and create strong chorio-retinal adhesions. RD with macula-on must be reattached within one week after macular detachment, otherwise visual recovery is progressively diminished [3].

\section{Complications}

Chronicity of RD causes PVR, hypotony, pigmentary glaucoma new iris vessels, cataracts and uveitis [3].

\section{Prognosis}

$5-15 \%$ of retinal re-attachment surgeries are unsuccessful [8].

\section{Imaging Presentations}

Figure $1 \& 2$

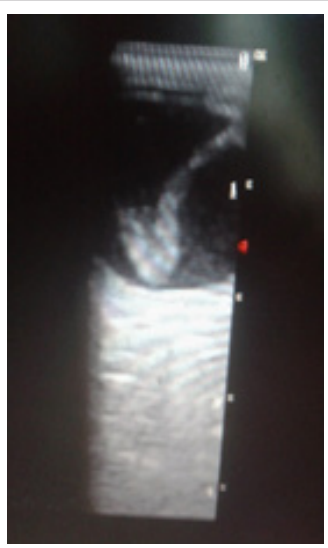

Figure 1: Coned view of B-scan echogram of an eye showing an intra-vitreous $\mathrm{V}$-shaped echogenic band of retinal detachment that is anchored posteriorly to the optic disc (arrow) and anteriorly to the ora serrata (curved arrow).

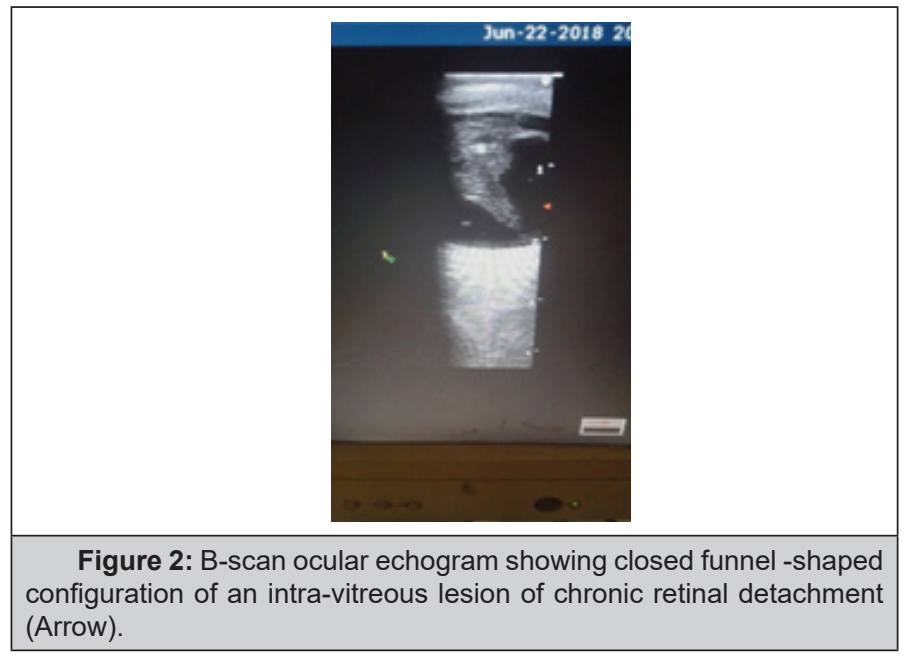

\section{Conclusion}

Retinal detachment is a potential blinding condition that can be readily detected with B-scan ophthalmic ultrasonography due to pathognomonic features.

\section{References}

1. Kang HK, Luff AJ (2008) Management of retinal detachment: a guide for non-opthalmologists. BMJ 336(7655): 1235-1240.

2. Bhende M, Gopal S, Gogi A, Sharma T, Gopal L, et al. (2013) The Sankara Nethralaya Atlas of Ophthalmic ultrasound. Retinal detachment. Jaypee Brothers, New Delhi, India, pp. 35-44.

3. Subhadra Jalali MS (2003) Retinal detachment. Comm Eye Health 16(46): 25-26.

4. Roka A, Rubero JA, Rosario J, Latha G (2019) Non-traumatic retinal detachment diagnosed via bedside ultrasonography. Cureus 10(6): e2771.

5. Mitry D, Charteris DG, Fleck BW, Campbell H, Singh J (2010) The epidermiology of rhegmatogenous retinal detachment geographical variation and clinical associations. Br J Ophthalmol 94(6): 678-684.

6. Chu HC, Chan MY, Chau CWJ (2017) The use of ocular ultrasound for the diagnosis of retinal detachment in a local accident and emergency department. Hong Kong J Emerg Med 24(6): 263-267.

7. Uduma FU, Akpan SI, Njeze NR (2019) B. scan ophthalmic ultrasonography: a review corroborated with echograms. New Frontiers in Opthalmology 5: 1-5.

8. Johnson Z, Ramsay A, Cottrell D, Mitchell K, Stannard K (2002) Triple cycle audit of primary retinal detachment surgery. Eye 16(3): 513-518. 